\title{
SIMULATION OF THE VIBRATIONS OF A NON-UNIFORM BEAM LOADED WITH BOTH A TRANSVERSELY AND AXIALLY ECCENTRIC TIP MASS
}

\author{
DESMOND ADAIR $^{1} \&$ MARTIN JAEGER $^{2}$ \\ ${ }^{1}$ School of Engineering, Nazarbayev University, Republic of Kazakhstan. \\ ${ }^{2}$ School of Engineering \& ICT, University of Tasmania, Australia.
}

\begin{abstract}
The main purpose of this work is to employ the Adomian modified decomposition method (AMDM) to calculate free transverse vibrations of non-uniform cantilever beams carrying a transversely and axially eccentric tip mass. The effects of the variable axial force are taken into account here, and Hamilton's principle and Timoshenko beam theory are used to obtain a single governing non-linear partial differential equation of the system as well as the appropriate boundary conditions. Two product non-linearities result from the analysis and the respective Cauchy products are computed using Adomian polynomials. The use of AMDM to make calculations for such a cantilever beam/tip mass arrangement has not, to the authors' knowledge, been used before. The obtained analytical results are compared with numerical calculations reported in the literature and good agreement is observed. The qualitative and quantitative knowledge gained from this research is expected to enable the study of the effects of an eccentric tip mass and beam non-uniformity on the vibration of beams for improved dynamic performance.

Keywords: Adomian decomposition method, cantilever beam, mechanical vibrations, tip mass
\end{abstract}

\section{INTRODUCTION}

Important components of engineering structures can be idealized as cantilever beams with a concentrated mass at its free end. Examples of this are antenna structures, large aspect aircraft wings fitted with external tanks or stores, flexible robot arms and atomic force microscopes. The presence of the tip mass plays an important role in the dynamic characteristics of the beam and exerts an inertial force, which is a function of the system motion [1]. The problem of a uniform cantilever beam with an eccentric tip mass has received much attention, for example, Prescott [2], Goel [3], and, Rama Bhat and Wagner [4]. Exact solutions have been found, for example, To [5] derived an exact expression for natural frequencies and mode shapes of a uniform beam with base excitation and an axially eccentric tip mass and Auciello [6] has suggested an exact analysis of free vibration of a linearly tapered cantilever beam with tip mass with rotary inertia and axial eccentricity. Work has been carried out for non-uniform beams which can achieve a better distribution of strength and weight. The non-uniformity, which usually arises from a variable beam cross section or inhomogeneous material properties, leads to a fourth-order partial differential equation with variable coefficients. In general these fourth-order equations cannot be solved analytically. However, for some specific non-uniform beams, exact solutions of the eigenvalue problem have been reported in terms of power series [7], orthogonal polynomials [8] and Bessel functions [9]. More recent work has been carried on beams with tip mass and with uniform [10] and non-uniform [1] cross sections. Matt [10] simulated transverse vibrations of a cantilever beam, with an eccentric tip mass, in the axial direction using integral transforms which included an implicit filter scheme. Malaeke and Moeenfard [1] modelled large amplitude flexural-extensional free vibrations of non-uniform beams carrying a transversely and axially eccentric tip mass with the effects of the variable axial force taken into account. 
The method of solution here is the Adomian modified decomposition method, which is a wide ranging method of solution of problems involving algebraic, differential [11], integro-differential [12] and partial differential equations [13]. Specific to this work, the Adomain decomposition and Adomian modified decomposition method have been used by several groups $[14,15]$ for uniform and non-uniform beams, starting with either the Euler-Bernoulli or Timoshenko formulations. Mao [14] applied the AMDM to rotating uniform beams and included a centrifugal stiffening term while Adair and Jaeger [15] applied the AMDM to rotating non-uniform beams which also included a centrifugal stiffening term. Yaman [16] has used the Adomian decomposition method to investigate the influence of the orientation effect on the natural frequency of a cantilever beam carrying a tip mass.

In this study we use the computational approach of AMDM to analyze free transverse vibrations of non-uniform cantilever beams carrying a transversely and axially eccentric tip mass. As the beam is assumed to be under the effect of gravity, the spatial variation of axial force is taken into account. The natural frequencies and mode shapes of the beam for three test cases were found and compared with results reported in the literature.

\section{MATHEMATICAL FORMULATION}

The schematic view of a non-uniform beam with an eccentric tip mass is shown in Fig. 1. The distances $a$ and $b$ are the transverse $(z)$ and axial ( $x$ ) eccentric values, respectively, $C o G$ is the centre of gravity of the tip mass $M$ and $l$ is the length of the beam. The polar mass moment of inertia of the tip mass around $C o G$ is $J$ and, in this analysis, the axial stretching of the beam due to both gravitational and inertial axial forces is considered.

In order the find the governing equations of motion of the beam, Hamilton's principle is employed. Every system behaves in such a way to satisfy

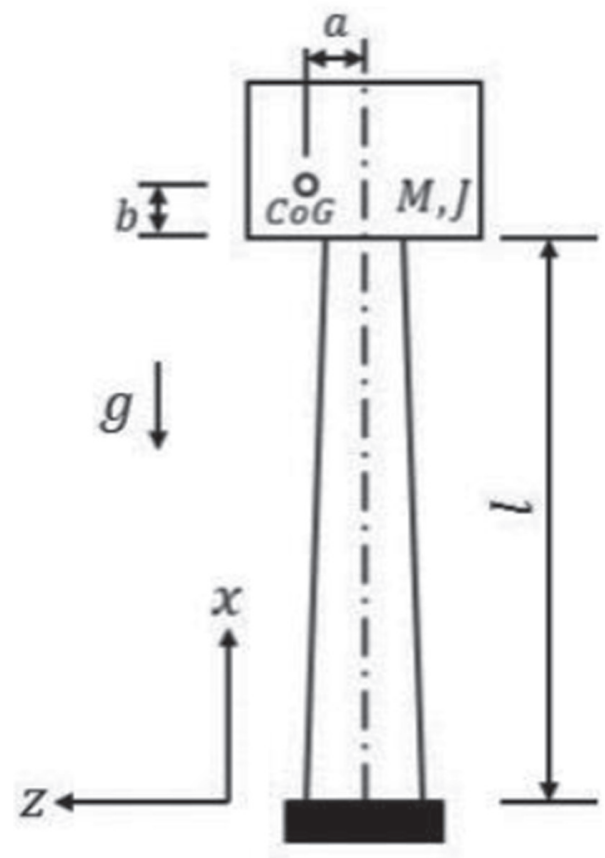

Figure 1: Schematic view of a non-uniform cantilever beam with an eccentric tip mass. 


$$
\delta \int_{t_{1}}^{t_{2}}\left(K-U+W_{e}\right) d t=0
$$

where $\delta$ is the variation operator, $t_{1}$ and $t_{2}$ are arbitrary times, $K$ and $U$ are the total kinetic and potential energies of the system respectively and $W_{e}$ is the work done by external forces.

For this system, the Euler-Bernoulli assumption that the rotation of cross-sections of the beam is small in comparison to the bending deformation is made. Also it is assumed that the angular distortion due to shear is considered small compared to the bending deformation and can be ignored. Therefore, the beam in question is long and slender with the length much greater than the thickness. With the assumption that the material of the beam is linear elastic, then the overall strain energy can be found from

$$
U=\frac{1}{2} \int_{0}^{l} E A(x)\left(\frac{\partial u}{\partial x}+\frac{1}{2}\left(\frac{\partial w}{\partial x}\right)^{2}\right)^{2} d x+\frac{1}{2} \int_{0}^{l} E I(x)\left(\frac{\partial^{2} w}{\partial x^{2}}\right)^{2} d x
$$

where $A(x)$ and $I(x)$ are the area and the second moment of area of the beam's cross-section around the neutral axis and $E$ is Young's modulus of elasticity of the beam's material.

The total kinetic energy of the system is given as $K=K_{\text {column }}+K_{\text {mass }}$, where

$$
\begin{gathered}
K_{\text {column }}=\frac{1}{2} \int_{0}^{l} \rho A(x)\left(\frac{\partial w}{\partial t}\right)^{2} d x+\frac{1}{2} \int_{0}^{l} \rho A(x)\left(\frac{\partial u}{\partial t}\right)^{2} d x \\
K_{\text {mass }}=\frac{1}{2} M V_{C o G}^{2}+\frac{1}{2} J_{C o G} \dot{\theta}^{2}=\frac{1}{2} M\left(\left(\frac{d w(l, t)}{d t}\right)^{2}+\left(a^{2}+b^{2}+r_{G}^{2}\right)\left(\frac{\partial^{2} w}{\partial x \partial t}(l, t)\right)^{2}\right. \\
\left.+2 b \frac{d w(l, t)}{d t} \frac{\partial^{2} w}{\partial x \partial t}(l, t)\right)+\frac{1}{2} M\left(\left(\frac{d u(l, t)}{d t}\right)^{2}-2 a \frac{d u(l, t)}{d t} \frac{\partial^{2} w}{\partial x \partial t}(l, t)\right) .
\end{gathered}
$$

Here, $\rho$ is the material density of the beam, $r_{G}$ is the radius of gyration of the tip mass, and $\dot{\theta}$ and $V_{C O G}$ are the rotational and translational velocities of the tip mass and its centre of gravity, respectively. Here $V_{C o G}$ is obtained using the relative velocity between the tip mass's centre of gravity and the point of attachment to the beam.

The virtual work done on the system with the assumption that no viscous damping is present is

$$
\delta W_{e}=\delta W_{g_{1}}+\delta W_{g_{2}},
$$

where $\delta W_{g_{1}}$ and $\delta W_{g_{2}}$ are the virtual gravitational work done on the tip mass and the beam, respectively. The virtual gravitational work done on the system can be written as

$$
\delta W_{g_{1}}=-M g \delta u(l, t)-M g a \delta\left(\frac{\partial w}{\partial x}(l, t)\right), \delta W_{g_{2}}=-\int_{0}^{l} g \rho A(x) \delta u(x, t) d x .
$$

Hamilton's principle is now applied to obtain the governing equations of vibration of the system 


$$
\begin{gathered}
\frac{\partial^{2}}{\partial x^{2}}\left(E I(x) \frac{\partial^{2} w(x, t)}{\partial x^{2}}\right)-\frac{\partial}{\partial x}\left(N(x, t) \frac{\partial w(x, t)}{\partial x}\right)+\frac{\partial}{\partial t}\left(\rho A(x) \frac{\partial w(x, t)}{\partial t}\right)=0 . \\
\frac{\partial}{\partial t}\left(\rho A(x) \frac{\partial u(x, t)}{\partial t}\right)-\frac{\partial N(x, t)}{\partial x}+g \rho A(x)=0
\end{gathered}
$$

The variable axial force of the beam, $N(x, t)$ is defined as

$$
N(x, t)=E A(x)\left(\frac{\partial u(x, t)}{\partial x}+\frac{1}{2}\left(\frac{\partial w(x, t)}{\partial x}\right)^{2}\right) .
$$

For a long and slender beam, with a small slenderness ratio, the longitudinal inertia is small compared to the restoring force and so

$$
\frac{\partial N(x, t)}{\partial x} \approx g \rho A(x)
$$

Substitution for the terms $N(x, t)$ and $\partial N(x, t) / \partial x$, as defined in eqns (9) and (10) into the expanded form of eqn (7) gives

$$
\begin{gathered}
{[E I(x)] \frac{\partial^{4} w(x, t)}{\partial x^{4}}+2[E I(x)]^{\prime} \frac{\partial^{3} w(x, t)}{\partial x^{3}}+[E I(x)]^{\prime \prime} \frac{\partial^{2} w(x, t)}{\partial x^{2}}-g \rho A(x) \frac{\partial w(x, t)}{\partial x}} \\
-E A(x)\left(\frac{\partial u(x, t)}{\partial x} \frac{\partial^{2} w(x, t)}{\partial x^{2}}+\frac{1}{2}\left(\frac{\partial w(x, t)}{\partial x}\right)^{2} \frac{\partial^{2} w(x, t)}{\partial x^{2}}\right)+\rho A(x) \frac{\partial^{2} w(x, t)}{\partial t^{2}}=0
\end{gathered}
$$

where the superscript ' mean differentiation w.r.t. $x$.

According to modal analysis for harmonic free vibration, $w(x, t)$ and $u(x, t)$, can be separable in space and so eqn (11) can be reduced to

$$
\begin{gathered}
{[E I(x)] \frac{\mathrm{d}^{4} w(x)}{\mathrm{d} x^{4}}+2[E I(x)]^{\prime} \frac{\mathrm{d}^{3} w(x)}{\mathrm{d} x^{3}}+[E I(x)]^{\prime \prime} \frac{\mathrm{d}^{2} w(x)}{\mathrm{d} x^{2}}-g \rho A(x) \frac{\mathrm{d} w(x)}{\mathrm{d} x}} \\
-E A(x)\left(\frac{\mathrm{d} u(x)}{\mathrm{d} x} \frac{d^{2} w(x)}{\mathrm{d} x^{2}}+\frac{1}{2}\left(\frac{\mathrm{d} w(x)}{\mathrm{d} x}\right)^{2} \frac{\mathrm{d}^{2} w(x)}{\mathrm{d} x^{2}}\right)-\rho A(x) \omega^{2} w(x)=0 .
\end{gathered}
$$

The dimensions of the beam in the $y$ and $z$ direction may be given as

$$
d(x)=d_{0}\left[1+\left(\alpha_{d}-1\right) \frac{x}{l}\right], d^{\prime}(x)=d_{0}^{\prime}\left[1+\left(a_{d^{\prime}}-1\right) \frac{x}{l}\right]
$$

where $\alpha_{d}=d_{1} / d_{0}, \alpha_{d^{\prime}}=d_{1}^{\prime} / d_{0}^{\prime} ; d_{0}, d_{0}^{\prime}$ are the cross-sectional widths at $x=0$ and $d_{1}, d_{1}^{\prime}$ are the cross-sectional widths at $x=l$. The area and moment of inertia of the beam vary according to 


$$
A(x)=A_{0}\left[1-\beta_{d^{\prime}} \frac{x}{l}\right]\left[1-\beta_{d} \frac{x}{l}\right], I(x)=I_{0}\left[1-\beta_{d^{\prime}} \frac{x}{l}\right]\left[1-\beta_{d} \frac{x}{l}\right]^{3},
$$

where $A_{0}=d_{0} d_{0}^{\prime}$ and $I_{0}=d_{0}^{\prime} d_{0}^{3} / 12$ are the cross-sectional area and moment of inertia at $x=0$, respectively, and $\beta_{d}=1-\alpha_{d}, \beta_{d^{\prime}}=1-\alpha_{d^{\prime}}$.

Without loss of generality, the following dimensionless quantities are now introduced.

$$
\begin{gathered}
\frac{w}{l} \rightarrow \phi, \frac{u}{l} \rightarrow \psi, \frac{x}{l} \rightarrow \eta, \frac{t}{T} \rightarrow \tau, \frac{a}{l} \rightarrow \bar{a}, \frac{b}{l} \rightarrow \bar{b}, \frac{r_{G}}{l} \rightarrow \bar{r}_{G}, \frac{g T^{2}}{l} \rightarrow \bar{g}, \frac{M}{l \rho A(0)} \rightarrow \bar{M}, \text { where } \\
T=l^{2} \sqrt{\frac{\rho A_{0}}{E I_{0}}} . \\
\lambda_{1}=\frac{E I(\eta)}{E I_{0}}, \lambda_{2}=\frac{A(\eta)}{A_{0}}, \lambda_{3}=\frac{E A(\eta) l^{2}}{E I_{0}}, \lambda_{4}=\frac{\rho A_{0} \omega^{2} l^{4}}{E I_{0}} \\
\lambda_{1}^{\prime}=\left[\frac{E I(\eta)}{E I_{0}}\right]^{\prime}, \lambda_{1}^{\prime \prime}=\left[\frac{E I(\eta)}{E I_{0}}\right]^{\prime \prime}
\end{gathered}
$$

Equation (12) can now be written in the form

$$
\begin{gathered}
\frac{\mathrm{d}^{4} \phi(\eta)}{\mathrm{d} \eta^{4}}+2 \frac{\lambda_{1}^{\prime}}{\lambda_{1}} \frac{d^{3} \phi(\eta)}{\mathrm{d} \eta^{3}}+\frac{\lambda_{1}^{\prime \prime}}{\lambda_{1}} \frac{\mathrm{d}^{2} \phi(\eta)}{\mathrm{d} \eta^{2}}-\bar{g} \frac{\lambda_{2}}{\lambda_{1}} \frac{\mathrm{d} \phi(\eta)}{\mathrm{d} \eta} \\
-\frac{\lambda_{3}}{\lambda_{1}}\left(\frac{\mathrm{d} \psi(\eta)}{\mathrm{d} \eta} \frac{\mathrm{d}^{2} \phi(\eta)}{\mathrm{d} \eta^{2}}+\frac{1}{2}\left(\frac{\mathrm{d} \phi(\eta)}{\mathrm{d} \eta}\right)^{2} \frac{\mathrm{d}^{2} \phi(\eta)}{\mathrm{d} \eta^{2}}\right)-\omega^{2} \frac{\lambda_{4}}{\lambda_{1}} \phi(\eta)=0 .
\end{gathered}
$$

The governing equation, eqn (17), cannot be solved as a closed-form solution and so some approximate approach must be adopted. Here the Adomian modified decomposition method (AMDM) is chosen.

\section{ADOMIAN MODIFIED DECOMPOSITION METHOD}

The Adomian decomposition method (ADM) is an iterative method, which has proved successful in treating non-linear equations. It is based on the search for a solution in the form of a series in which the non-linear terms are calculated recursively using the Adomian polynomials. The ADM gives the solutions $\phi(\eta)$ of eqn (17) in a series form of the infinite sum

$$
\phi(\eta)=\sum_{j=0}^{+\infty} \phi_{j}(\eta)
$$

Substitution of eqn (18) into the linear and nonlinear terms of eqn (17) yields

$$
L \sum_{j=0}^{+\infty} \phi_{j}(\eta)+R \sum_{j=0}^{+\infty} \phi_{j}(\eta)-\frac{\lambda_{3}}{\lambda_{1}} N_{1}[\psi, \phi]-\frac{1}{2} \frac{\lambda_{3}}{\lambda_{1}} N_{2}[\phi]=0,
$$


where

$$
L=\frac{\mathrm{d}^{4}}{\partial \eta^{4}}, R=\frac{\lambda_{1}^{\prime}}{\lambda_{1}} \frac{\partial^{3}}{\partial \eta^{3}}+\frac{\lambda_{1}^{\prime \prime}}{\lambda_{1}} \frac{\partial^{2}}{\partial \eta^{2}}-\bar{g} \frac{\lambda_{2}}{\lambda_{1}} \frac{\partial}{\partial \eta}-\omega^{2} \frac{\lambda_{2}}{\lambda_{1}} .
$$

Here $L$ is an invertible operator, which is taken as the highest-order derivative and $R$ is the remainder of the linear operator.

$N_{1}[\psi, \phi]$ in eqn (19) is a product nonlinearity and the respective Cauchy product can be computed using Adomian polynomials $\left(A_{n}\right)$ as

$$
\begin{aligned}
N_{1}[\psi, \phi]= & \sum_{j=0}^{+\infty} A_{j}\left(\psi_{0}, \ldots, \psi_{j} ; \phi_{0}, \ldots, \phi_{j}\right)= \\
& \frac{\partial \psi}{\partial \eta} \frac{\partial^{2} \phi}{\partial \eta^{2}}=\sum_{j=0}^{+\infty} \frac{\partial \psi_{n}}{\partial \eta} \sum_{m=0}^{+\infty} \frac{\partial^{2} \phi_{m}}{\partial \eta^{2}}=\sum_{j=0}^{+\infty} \sum_{m=0}^{j} \frac{\partial \psi_{m}}{\partial \eta} \frac{\partial^{2} \phi_{j-m}}{\partial \eta^{2}} .
\end{aligned}
$$

$N_{2}[\phi]$ in eqn (19) is also a product non-linearity, and accordingly the respective Cauchy product can be computed using Adomian polynomials $\left(B_{n}\right)$ as

$$
\begin{aligned}
N_{2}[\phi]= & \sum_{j=0}^{+\infty} B_{j}\left(\phi_{0}, \ldots, \phi_{j}\right)=\left(\frac{\partial \phi}{\partial \eta}\right)^{2} \frac{\partial^{2} \phi}{\partial \eta^{2}}=\frac{\partial^{2} \phi}{\partial \eta^{2}}\left(\frac{\partial \phi}{\partial \eta}\right)^{2}= \\
& \sum_{j=0}^{+\infty} \frac{\partial^{2} \phi_{n}}{\partial \eta^{2}} \sum_{m=0}^{+\infty} \frac{\partial \phi_{m}}{\partial \eta} \sum_{l=0}^{+\infty} \frac{\partial \phi_{l}}{\partial \eta}=\sum_{j=0}^{+\infty} \frac{\partial^{2} \phi_{n}}{\partial \eta^{2}} \sum_{m=0}^{+\infty} \sum_{l=0}^{+\infty} \frac{\partial \phi_{l}}{\partial \eta} \frac{\partial \phi_{m-l}}{\partial \eta}= \\
& \sum_{j=0}^{+\infty} \sum_{m=0}^{j} \frac{\partial^{2} \phi_{m}}{\partial \eta^{2}} \sum_{l=0}^{j-m} \frac{\partial \phi_{l}}{\partial \eta} \frac{\partial \phi_{j-m-l}}{\partial \eta} .
\end{aligned}
$$

According to the Adomian modified decomposition method (AMDM) the terms $\phi_{j}(\eta)$ in eqn (18) can be expressed as $C_{j} \eta^{j}$ so giving the infinite series

$$
\phi(\eta)=\sum_{j=0}^{+\infty} C_{j} \eta^{j}
$$

where the unknown coefficients $C_{j}$ are determined concurrently. Using a similar approach $\psi(\eta)$ can also be equated to the series $\sum_{j=0}^{+\infty} C_{j}^{\prime} \eta^{j}$.

Using the linear operator $L$ of Eq. (20) then the inverse operator of $L$ is a 4-fold integral operator defined as

$$
L^{-1}=\int_{0}^{\eta} \int_{0}^{\eta} \int_{0}^{\eta} \int_{0}^{\eta}(\cdots) \mathrm{d} \eta \mathrm{d} \eta \mathrm{d} \eta \mathrm{d} \eta
$$

Equation (19) can be written as

$$
\phi(\eta)=\Phi(\eta)-L^{-1}\left[R \sum_{j=0}^{+\infty} C_{j} \eta^{j}+\frac{\lambda_{3}}{\lambda_{1}} \sum_{j=0}^{+\infty} A_{j} \eta^{j}+\frac{1}{2} \frac{\lambda_{3}}{\lambda_{1}} \sum_{j=0}^{+\infty} B_{j} \eta^{j}\right],
$$


where $R$ is now

$$
\begin{aligned}
R=\frac{\lambda_{1}^{\prime}}{\lambda_{1}} \sum_{j=0}^{+\infty}(j+1)(j+2)(j+3) C_{j+3} & \eta^{j}+\frac{\lambda_{1}^{\prime \prime}}{\lambda_{1}} \sum_{j=0}^{\infty}(j+1)(j+2) C_{j+2} \eta^{j} \\
& -\bar{g} \frac{\lambda_{2}}{\lambda_{1}} \sum_{j=0}^{\infty}(j+1) C_{j+1} \eta^{j}-\omega^{2} \frac{\lambda_{4}}{\lambda_{1}} \sum_{j=0}^{\infty} C_{j} \eta^{j},
\end{aligned}
$$

The Adomian's polynomials in eqn (25) are found using the standard method [17] for $B_{j}\left(\phi_{0}, \ldots, \phi_{j}\right)$ and the generalized method for several variables as reported by Adomian and Rach [18] is used for $A_{j}\left(\psi_{0}, \ldots, \psi_{j} ; \phi_{0}, \ldots, \phi_{j}\right)$.

The term $\Phi(\eta)$ found in eqn (25) is the initial term and defined as

$$
\Phi(\eta)=\sum_{j=0}^{3} C_{j} \eta^{j}=\phi(0)+\phi^{\prime}(0) \eta+\phi^{\prime \prime}(0) \eta^{2} / 2+\phi^{\prime \prime \prime}(0) \eta^{3} / 6
$$

On substituting eqns (23), (26) and (27) into eqn (25), the following is obtained

$$
\begin{aligned}
& \sum_{j=0}^{\infty} C_{j} \eta^{j}=\phi(0)+\phi^{\prime}(0) \eta+\phi^{\prime \prime}(0) \eta^{2} / 2+\phi^{\prime \prime \prime}(0) \eta^{3} / 6 \\
& -\sum_{j=0}^{\infty}\left\{\frac{\eta^{j+4}}{(j+1)(j+2)(j+3) j+4)}\right. \\
& \sum_{m=0}^{j}\left[(m+1)(m+2)(m+3) \frac{\lambda{ }_{1}}{\lambda_{1}} C_{m+3}+(m+1)(m+2) \frac{\lambda^{\prime \prime}}{\lambda_{1}} C_{m+2}\right. \\
& \left.\left.-(m+1) \bar{g} \frac{\lambda_{2}}{\lambda_{1}} C_{m+1}-\omega^{2} \frac{\lambda_{4}}{\lambda_{1}} C_{m}\right]+\frac{\lambda_{3}}{\lambda_{1}}\left(A_{j}+\frac{1}{2} B_{j}\right)\right\}
\end{aligned}
$$

The recurrence relation for the coefficients $C_{j}$ can now be stated and the solution for $\phi(\eta)$ can be calculated from eqn (28). The series solution is $\phi(\eta)=\sum_{j=0}^{\infty} C_{j} \eta^{j}$, although all of the coefficients $C_{j}$ cannot yet be determined, and thus, the solutions must be approximated by the truncated series $\sum_{j=0}^{n-1} C_{j} \eta^{j}$, with the successive approximations being $\phi^{[n]}(\eta)=\sum_{j=0}^{n-1} C_{j} \eta^{j}$ as $n$ increases and the boundary conditions are met.

Thus $\phi^{[1]}(\eta)=C_{0}, \phi^{[2]}(\eta)=\phi^{[1]}(\eta)+C_{1} \eta, \phi^{[3]}=\phi^{[2]}(\eta)+C_{2} \eta^{2}$ serve as approximate solutions with increasing accuracy as $n \rightarrow \infty$. The three coefficients $C_{j}(j=0,1,2,3)$ depend on the boundary equations. In this case, the two coefficients $C_{0}$ and $C_{1}$ can be chosen as arbitrary constants, and the other two coefficients $C_{2}$ and $C_{3}$ are stated in terms of the problem parameters or as the functions of the other coefficients.

The initial term $\Phi(\eta)$ in eqn (25) is a function of $C_{0}$ and $C_{1}$ and from the recurrence relation obtained from eqn (28) the coefficients $C_{j}(j \geq 4)$ are functions of $C_{0}$ and $C_{1}$ and $\lambda_{4}$. By substituting $\phi^{[n]}(\eta)$ into the boundary conditions we then have 


$$
f_{r 0}^{[n]}\left(\lambda_{4}\right) C_{0}+f_{r 1}^{[n]}\left(\lambda_{4}\right) C_{1}=0, r=1,2 .
$$

For non-trivial solutions, $C_{0}$ and $C_{1}$, the frequency equation is given a

$$
\left|\begin{array}{ll}
f_{10}^{[n]}\left(\lambda_{4}\right) & f_{11}^{[n]}\left(\lambda_{4}\right) \\
f_{20}^{[n]}\left(\lambda_{4}\right) & f_{21}^{[n]}\left(\lambda_{4}\right)
\end{array}\right|=0
$$

The $i$ th estimated eigenvalue $\lambda_{2(i)}^{[n]}$ corresponding to $n$ is obtained from eqn (30), i.e. the $i$ th estimated dimensionless natural frequency $\Omega_{n(i)}^{[n]}=\sqrt{\lambda_{2(i)}^{[n]}}$ is also obtained and $n$ is determined by the following equation

$$
\left|\Omega_{n(i)}^{[n]}-\Omega_{n(i)}^{[n-1]} \leq \varepsilon\right|
$$

where $\Omega_{n(i)}^{[n-1]}$ is the $i$ th estimated dimensionless natural frequency corresponding to $n-1$, and $\varepsilon$ is a preset sufficiently small value. If eqn (31) is satisfied, then $\Omega_{n(i)}^{[n]}$ is the $i$ th dimensionless natural frequency $\Omega_{n(i)}$. By substituting $\Omega_{n(i)}^{[n]}$ into eqn (29)

$$
C_{1}=-\frac{f_{r 0}^{[n]}\left(\Omega_{n(i)}^{[n]}\right)}{f_{r 1}^{[n]}\left(\Omega_{n(i)}^{[n]}\right)} C_{0}, r=1 \text { or } 2 .
$$

and all of the other coefficients $C_{j}$ can be obtained from recurrence relations. Furthermore, the $i$ th mode shape $\phi_{i}^{[n]}$ corresponding to the $i$ th eigenvalue $\Omega_{n(i)}^{[n]}$ is obtained by

$$
\phi_{i}^{[n]}(\eta)=\sum_{j=0}^{n-1} C_{j}^{[i]} \eta^{j}
$$

where $C_{j}^{[i]}(\eta)$ is $C_{j}(\eta)$ in which $\lambda_{4}$ is substituted by $\lambda_{4(i)}$ and $\phi_{i}^{[n]}$ is the $i$ th eigenfunction corresponding to the $i$ th eigenvalue $\lambda_{4(i)}$. By normalizing eqn (33) the $i$ th normalized eigenfunction is defined as

$$
\bar{\phi}_{i}^{[n]}(\eta)=\frac{\phi_{i}^{[n]}(\eta)}{\sqrt{\int_{0}^{1}\left[\phi_{i}^{[n]}(\eta)\right]^{2} d \eta}}
$$

where $\bar{\phi}_{i}^{[n]}(\eta)$ is the $i$ th mode shape function of the beam corresponding to the $i$ th natural frequency $\omega_{i}^{[n]}=\sqrt{\lambda_{i}^{[n]}} \sqrt{E I_{0} / \rho A l^{4}}={ }_{n(i)}^{[n]} \sqrt{E I_{0} / \rho A l^{4}}$.

\section{RESULTS}

Results are given for the calculations of natural frequencies and mode shapes for a non-uniform beam, a uniform beam with constant axial load, i.e. the non-linear terms of eqn (17) were made constant, and an eccentric mass, and finally, a non-uniform beam with an eccentric mass. The calculated results are compared with results found in the literature. 


\subsection{Case 1: non-uniform beam}

The test case present here is a clamped-free constant width tapered beam (wedge) and the boundary conditions used were

$$
\phi(0)=0, \frac{\mathrm{d} \phi(0)}{\mathrm{d} \eta}=0, \frac{\mathrm{d}^{2} \phi(1)}{\mathrm{d} \eta^{2}}=0, \frac{\mathrm{d}^{3}(1)}{\mathrm{d} \eta^{3}}=0 .
$$

For the wedge beam $\alpha_{d^{\prime}}=1, \alpha_{d}=\alpha ; \beta_{d^{\prime}}=0, \beta_{d}=\beta$ the area and moment of the section can now be written as

$$
A(\eta)=A_{0}[1+(\alpha-1) \eta]=A_{0}(1-\beta \eta), I(\eta)=I_{0}[1+(\alpha-1) \eta]^{3}=I_{0}(1-\beta \eta)^{3}
$$

where $\beta$ is known as the taper ratio.

In this case the coefficients $C_{0}$ and $C_{1}$ were set to zero and the coefficients $C_{2}$ and $C_{3}$ are arbitrary constant. By substituting $\phi^{[n]}(\eta)=\sum_{j=0}^{n-1} C_{j} \eta^{j}$ into the last two boundary conditions of eqn (35) the following algebraic equations involving $C_{2}$ and $C_{3}$ are obtained

$$
\begin{aligned}
& \sum_{j=0}^{n-3}(j+1)(j+2) C_{j+2}=f_{12}^{[n]}\left(\lambda_{4}\right) C_{2}+f_{13}^{[n]}\left(\lambda_{4}\right)=0, \\
& \sum_{j=0}^{n-4}(j+1)(j+2)(j+3) C_{j+3}=f_{22}^{[n]}\left(\lambda_{4}\right) C_{2}+f_{23}^{[n]}\left(\lambda_{4}\right) C_{3}=0 .
\end{aligned}
$$

and for non-trivial solutions of $C_{2}$ and $C_{3}$ the frequency equation can be written as

$$
f_{12}^{[n]}\left(\lambda_{4}\right) f_{23}^{[n]}\left(\lambda_{4}\right)-f_{22}^{[n]}\left(\lambda_{4}\right) f_{13}^{[n]}\left(\lambda_{4}\right)=0
$$

By solving eqn (37) for $n$ and taking the real root for $\lambda_{4}=\Omega_{n}^{2}$, it was found that for $n=30$

$$
\mid \Omega_{n(1)}^{[30]}-\Omega_{n(1)}^{[29]} \leq \varepsilon=0.00001 .
$$

with $\Omega_{n(1)} \approx \Omega_{n(1)}^{[30]}=3.517$.

The results for the wedge beam for different taper ratios are compared with those reported by Banerjee et al. [19], as shown in Table 1. The calculated results are in reasonable agreement with those reported by Banerjee et al. [19]. Values for $\beta=0$ are not reported by Banerjee et al. [19] as their method suffered numerical overflow when $\beta=0$.

Reasonable agreement was found between the two methods, although the current calculations generally gave slightly larger values. The maximum difference between the two methods was of the order of $1 \%$ and occurred for $\Omega_{n(3)}$.

4.2 Case 2: Uniform beam with eccentric tip mass experiencing constant axial force

Following [1] calculations were made for a uniform beam experiencing a constant axial loading with an eccentric tip mass. The material and geometric properties are listed in Table 2. The boundary conditions used for these calculations and those for Case 3 are 
Table 1: Normalized natural frequencies of a wedge beam.

\begin{tabular}{lllllll}
\hline$\beta$ & $\Omega_{n(1)}$ & \multicolumn{5}{c}{$\Omega_{n(2)}$} \\
\hline & Current & Ref.[21] & Current & Ref.[21] & Current & Ref.[21] \\
\hline 0 & 3.517 & - & 22.100 & - & 61.003 & - \\
0.1 & 3.562 & 3.559 & 21.346 & 21.338 & 59.001 & 58.980 \\
0.2 & 3.612 & 3.608 & 20.639 & 20.621 & 56.293 & 56.192 \\
0.3 & 3.671 & 3.667 & 19.905 & 19.881 & 53.592 & 53.322 \\
0.5 & 3.830 & 3.824 & 18.355 & 18.317 & 47.482 & 47.265 \\
0.7 & 4.090 & 4.082 & 16.667 & 16.625 & 41.005 & 40.588 \\
0.9 & 4.641 & 4.631 & 14.987 & 14.931 & 33.231 & 32.833 \\
\hline
\end{tabular}

Table 2: Properties of the uniform beam with tip mass.

\begin{tabular}{lllllllll}
\hline Parameter & $E$ & $\rho$ & $M$ & $l$ & $a$ & $b$ & $r_{G}$ & $D$ \\
\hline Value & 210 & 7680 & 5000 & 30 & 1 & 1 & 0.5 & 3 \\
& $\mathrm{GPa}$ & $\mathrm{kg} / \mathrm{m}^{3}$ & $\mathrm{~kg}$ & $\mathrm{~m}$ & $\mathrm{~m}$ & $\mathrm{~m}$ & $\mathrm{~m}$ & $\mathrm{~m}$ \\
\hline
\end{tabular}

$$
\phi(0)=0, \frac{\mathrm{d} \phi(0)}{\mathrm{d} \eta}=0, \frac{\mathrm{d}^{2} \phi(1)}{\mathrm{d} \eta^{2}}=-\frac{\bar{M} \overline{g a}}{\lambda_{1}}, \lambda_{1} \frac{\mathrm{d}^{3} \phi(1)}{\mathrm{d} \eta^{3}}+\lambda_{1}^{\prime} \frac{\mathrm{d}^{2} \phi(1)}{\mathrm{d} \eta^{2}}+\bar{M} \bar{g} \frac{\mathrm{d} \phi(1)}{\mathrm{d} \eta}=0
$$

An exact solution for this eigenvalue problem can be found [1] and is used here for comparison.

It can be seen form Table 2 that generally the current calculations give a closer result to the exact solution.

\subsection{Case 3: Non-uniform beam with eccentric tip mass and rotary inertia}

Here a linearly varying cross section cantilever beam with a both transversely and axially eccentric tip mass with rotary inertia is considered. The beam here is tubular and the physical properties are given in Table 4.

Using the present method, the normalized natural frequencies and mode shapes of the beam are obtained as on Fig. 2.

Table 3: Normalized natural frequencies of uniform beam.

\begin{tabular}{lllll}
\hline$N$ & $\Omega_{n(1)}$ & $\Omega_{n(2)}$ & $\Omega_{n(3)}$ \\
\hline 0 & Exact & 0.7670 & 4.7763 & 13.2822 \\
& Present & 0.7678 & 4.7764 & 13.2955 \\
& FEA Ref. [1] & 0.7675 & 4.7707 & 13.2460 \\
$10^{5}$ & Exact & 0.7657 & 4.7749 & 13.2811 \\
& Present & 0.7663 & 4.7763 & 13.2965 \\
& FEA Ref. [1] & 0.7662 & 4.7693 & 13.2451 \\
\hline
\end{tabular}


Table 4: Physical and geometrical properties of non-uniform beam with eccentric tip mass.

\begin{tabular}{lllllllll}
\hline Parameter & $E$ & $\rho$ & $M$ & $l$ & $\beta$ & $a$ & $b$ & $r_{G}$ \\
\hline Value & 210 & 7680 & 5000 & 30 & 0.8 & 0.5 & 0.5 & 0.3 \\
& GPA & $\mathrm{kg} / \mathrm{m} 3$ & $\mathrm{~kg}$ & $\mathrm{~m}$ & & $\mathrm{~m}$ & $\mathrm{~m}$ & $\mathrm{~m}$ \\
\hline
\end{tabular}

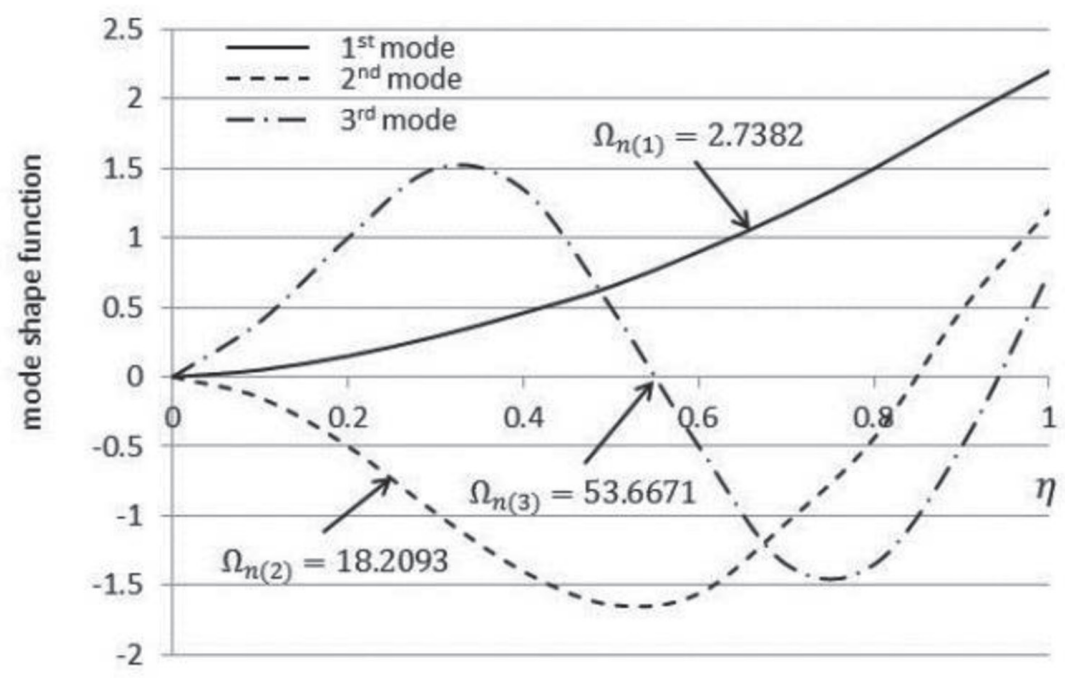

Figure 2: First three normalized natural frequencies and mode shapes for Case 3.

\section{CONCLUSIONS}

A method has been developed for calculating characteristics of a vibrating non-uniform cantilever beam with an eccentric tip mass. The developed approach is nontrivial and complicated due to the nature of the problem. No exact solution of this general problem is available. Several test cases were performed with quite reasonable agreement between the results of the current approach and those reported in the literature found.

\section{REFERENCES}

[1] Malaeke, H. \& Moeenfard, H., Analytical modeling of large amplitude free vibration of non-uniform beams carrying a both transversely and axially eccentric tip mass. Journal of Sound and Vibration, 366, pp. 211-229, 2016.

https://doi.org/10.1016/j.jsv.2015.12.003

[2] Prescott, J., Applied elasticity, Dover Publications, New York, 1946.

[3] Goel, R., Vibrations of a beam carrying a concentrated mass. Journal of Applied Mechanics, 40, pp. 821-822, 1973.

https://doi.org/10.1115/1.3423102

[4] Rama Bhat, B., \& Wagner, H., Natural frequencies of a uniform cantilever with a tip mass slender in the axial direction. Journal of Sound and Vibration, 45(2), pp. 304-307, 1976. https://doi.org/10.1016/0022-460x(76)90606-4

[5] To, C.W.S., Vibration of a cantilever beam with a base excitation and tip mass. Journal of Sound and Vibration, 172, pp. 289-304, 1994. 
[6] Auciello, N.M., Transverse vibration of a linearly tapered cantilever beam with tip mass of rotary inertia and eccentricity. Journal of Sound and Vibration, 194, pp. 25-34, 1996. https://doi.org/10.1006/jsvi.1996.0341

[7] Mable, H. \& Rogers, C., Transverse vibrations of double-tapered cantilever beams with end support and with end mass. The Journal of the Acoustical Society of America, 55, pp. 986-991, 1974. https://doi.org/10.1121/1.1914673

[8] Caruntu, D., On bending vibrations of some kinds of variable cross-section using orthogonal polynomials. Revue Roumaine des Sciences Techniques-Série Délelött Mécanique Appliquée, 41, pp. 265-272, 1996.

[9] Sanger, D., Transverse vibration of a class of non-uniform beams. Journal of Mechanical Engineering Science, 10, pp. 111-120, 1968.

https://doi.org/10.1243/jmes_jour_1968_010_018_02

[10] Matt, C. F. T., Simulation of the transverse vibrations of a cantilever beam with an eccentric tip mass in the axial direction using integral transforms. Applied Mathematical Modelling, 37, pp. 9338-9354, 2013.

https://doi.org/10.1016/j.apm.2013.04.038

[11] Adomian, G., Nonlinear stochastic system theory and application to physics. Kluwer Academic, Dordrecht, 1988.

[12] Adomian, G. \& Rach, R., On composite nonlinearities and the decomposition method. Journal of Mathematical Analysis and Applications, 113(2), pp. 504-509, 1986. https://doi.org/10.1016/0022-247x(86)90321-5

[13] Wazwaz, A. M., Analytic treatment for variable coefficient fourth-order parabolic partial differential equations. Applied Mathematics and Computation, 123, pp. 219-227, 2001. https://doi.org/10.1016/s0096-3003(00)00070-9

[14] Mao, Q., Application of Adomain modified decomposition method to free vibration analysis of rotating beams. Mathematical Problems in Engineering, 2013, pp. 1-10, 2013.

https://doi.org/10.1155/2013/284720

[15] Adair, D. \& Jaeger, M., Simulation of tapered rotating beams with centrifugal stiffening using the Adomian decomposition method. Applied Mathematical Modelling, 40, pp. 3230-3241, 2016.

https://doi.org/10.1016/j.apm.2015.09.097

[16] Yaman, M., Adomian decomposition method for solving a cantilever beam of varying orientation with tip mass. Journal of Computational and Nonlinear Dynamics, 2 , pp. 52-57, 2007. https://doi.org/10.1115/1.2389167

[17] Adomian, G., A new approach to nonlinear partial differential equations. Journal of Mathematical Analysis and Applications, 102(2), pp. 420-434, 1984. https://doi.org/10.1016/0022-247x(84)90182-3

[18] Adomian, G. \& Rach, R., Generalization of Adomian polynomials to functions of several variables. Computers \& Mathematics with Applications, 24(5/6), pp. 11-24, 1992. https://doi.org/10.1016/0898-1221(92)90037-i

[19] Banerjee, J. R., Su, H. \& Jackson, D. R., Free vibration of rotating tapered beams using the dynamic stiffness method. Journal of Sound and Vibration, 298, pp. 1034-1054, 2006. https://doi.org/10.1016/j.jsv.2006.06.040 